\title{
Treatment with oxygen-ozone therapy in hemodialysis patients
}

\author{
Giuseppe Bonforte, ${ }^{1}$ Hilary Riva, ${ }^{1}$ Olivier Giannini, ${ }^{2}$ Brenno Balestra ${ }^{2}$ \\ ${ }^{1}$ Nephrology Unit, Beata Vergine Hospital, Mendrisio; ${ }^{2}$ Department of Internal Medicine, Beata Vergine Hospital, \\ Mendrisio, Switzerland
}

\begin{abstract}
Peripheral arterial disease and secondary amyloidosis account for significant morbidity and mortality among uremic patients. Several papers demonstrated the beneficial effects of ozone therapy in the treatment of vascular and orthopaedic diseases. The aim of this paper is to study these effects among hemodialysed patients with severe complications. Seventy-five hemodialysed patients were studied. Sixty patients suffered from peripheral arterial occlusive disease. Twenty-three of them underwent previous unilateral lower limb amputation (Group 1). Fifteen subjects had remarkable joint pain due to a2-microglobulin deposition (Group 2). All patients were treated with analgesic therapy. The Italian Society for Oxygen-Ozone Therapy (SIOOT) protocols were applied: the patients received major $\mathrm{O}_{3}$ autohemotherapy, subcutaneous ozone infusion and body exposure to oxygen/ozone mixture by bagging. A questionnaire was submitted to the patients at the beginning and at the end of ozone therapy for the individual assessment of treatment efficacy. Complete healing of the ischemic ulcers was obtained in many patients. Eight-eight percent of the patients reported a subjective successful outcome after ozone therapy. Both Group 1 and Group 2 subjects reported a significant decrease in pain perception. The use of analgesic drugs was reduced in $78 \%$ of them and $72 \%$ got a normal sleep/wake cycle. An enhancement in physical
\end{abstract}

Correspondence: Giuseppe Bonforte, Nephrology Unit, Beata Vergine Hospital, Via Alfonso Turconi 23, Mendrisio 6850, Switzerland. E-mail: giuseppe.bonforte@gmail.com

Key words: Oxygen-ozone therapy; Dialysis, Peripheral vascular disease; Lower limb ischemia; Gangrene; b2-microglobulin; Secondary amyloidosis.

Conflict of interest: the authors declare no potential conflict of interest.

Funding: none.

Received for publication: 24 June 2019.

Accepted for publication: 5 July 2019.

${ }^{\circ}$ Copyright: the Author(s), 2019

Licensee PAGEPress, Italy

Ozone Therapy 2019; 4:8376

doi:10.4081/ozone.2019.8376

This article is distributed under the terms of the Creative Commons Attribution Noncommercial License (by-nc 4.0) which permits any noncommercial use, distribution, and reproduction in any medium, provided the original author(s) and source are credited. activity was reported by $67 \%$ of the subjects. Ozone therapy improves hemodialysed patients' quality of life reducing bone pain perception and attenuating clinical signs of peripheral vascular disease.

\section{Introduction}

Ozone therapy has been known for more than fifty years. Several papers have confirmed the beneficial effects of oxygenozone therapy in the treatment of vascular disorders, ${ }^{1-3}$ osteoarthritis, ${ }^{4,5}$ orthopedic pathology 6 and various painful syndromes., ${ }^{4,5}$ Ozone therapy is commonly administered through minor $\mathrm{O}_{3}$ autohemotherapy and major $\mathrm{O}_{3}$ autohemotherapy. ${ }^{7}$ The precise control of ozone dosage is mandatory in order to avoid toxic side-effects.

Patients with end-stage renal disease undergoing dialysis have a high risk of developing peripheral arterial occlusive disease ${ }^{8}$ and secondary amyloidosis. ${ }^{9}$ Lower limb amputation, ${ }^{10}$ carpal tunnel syndrome, remarkable pain in the shoulders and in the cervical and lumbar column are common in subjects with a long history of dialysis. The optimal management of these complications in endstage renal disease (ESRD) patients is quite controversial. Thus we performed a prospective study evaluating the impact of ozonotherapy on peripheral arterial disease and pain in uremic patients undergoing hemodialysis.

\section{Materials and Methods}

Seventy-five uremic hemodialysis patients (41 female, 34 male), mean age 73 years (range 48-97) with end stage renal disease treated with hemodialysis for 13 years (range 4-34). Sixty patients suffered from stage II b-IV peripheral arterial occlusive disease according to the Fontain score (twenty-three of them had previous unilateral lower limb amputation, Group 1). Fifteen subjects had remarkable joint pain due to a2-microglobulin deposition (Group 2).

In Group 1 the causes of ESRD were diabetic nephropathy in 24 patients and ischemic nephropathy in 36 patients. In Group 2 renal diseases consisted in chronic pyelonephritis ( $8 \mathrm{pts}$ ); ischemic nephropathy (2 patients) and chronic glomerulonephritis (5 patients).

All patients underwent three times a week hemodiafiltration or bicarbonate hemodialysis treatment and all of them were taking analgesics).

The hemodialysis adequacy ${ }^{11}$ and the hemoglobin levels were evaluated monthly.

Ozone generator (MULTIOSSIGEN Medical 95 
Computerised Photometric System, Gorle, Bergamo, Italy) was used in the study. The Italian Society for Oxygen-Ozone Therapy (SIOOT) protocols were applied. ${ }^{12}$ Group 1 received major $\mathrm{O}_{3}$ autohemotherapy $(200 \mathrm{~mL}$ of blood in association with $200 \mathrm{cc}$ of an oxygen/ozone mixture at a concentration of 20-40 $\mathrm{mcg} / \mathrm{mL}$ ), ischemic leg exposure to ozone by bagging (oxygen/ozone mixture at a concentration of 3-30 mcg for 10 min; Figure 1) and subcutaneous ozone infusion around the ischemic ulcers (50 cc of oxygen/ozone mixture at a concentration of $3 \mathrm{mcg} / \mathrm{mL}$ ); these procedures were performed twice a week for at least 8 weeks.

In sixteen patients the treatment was repeated more than once at a distance of time.

Five patients with extremely severe peripheral ulcerative lesions were treated with patient's own blood mixed with ozone (minor $\mathrm{O}_{3}$ autohemotherapy) to promote wound healing (Figure 2).

Group 2 received major $\mathrm{O}_{3}$ autohemotherapy $(200 \mathrm{~mL}$ of blood in association with $200 \mathrm{cc}$ of an oxygen/ozone mixture with a concentration of $40 \mathrm{mcg} / \mathrm{mL}$ ) and subcutaneous ozone infiltration in the painful areas such as shoulder, knee, wrist and/or vertebral column (25-50 cc of oxygen/ozone mixture at a concentration of 2 to $10 \mathrm{mcg} / \mathrm{mL}$ ); these procedures were performed twice a week for at least 1 month and then every seven or fifteen days according to patient's response.

A questionnaire for individual assessment of the treatment efficacy was submitted at the beginning and at the end of ozone therapy: the parameters evaluated were pain, measured with a $0-10$ point Visual Analog Scale, exercise ability and analgesic drugs consumption.

The changes of continuous variables were assessed by ANOVA for repeated measurements. $\mathrm{P}<0.05$ was considered as significant. Statistical analysis was performed using Statview software (5.0 for Windows).

\section{Results}

All patients completed the protocol without side effects. Hemodialysis adequacy and hemoglobin levels remained stable during the study.

In the individual assessment of treatment efficacy, $88 \%$ of them reported a successful outcome after ozone therapy: 48 patients experienced optimal improvement ( 8 secondary amyloidosis; 22 IIb stage peripheral ischemic disease, 6 III stage peripheral ischemic disease and 12 IV stage peripheral ischemic disease), 19 patients reported a significant improvement (5 secondary amyloidosis; 14 patients with peripheral ischemic disease) and 8 patients observed no changes compared to baseline $(2 \mathrm{sec}-$ ondary amyloidosis and 6 patients with peripheral ischemic disease; Table 1).

Both Group 1 and Group 2 subjects reported a significant decrease in pain perception (Figure 3; $\mathrm{P}<0.001$ ).

Furthermore, 60 patients reduced the prescribed dose of analgesic drugs or discontinued their assumption, instead 16 patients maintained the same dose of therapy; $72 \%$ of patients showed a normal sleep/wake cycle.

An enhancement in exercise activity was reported in $67 \%$ of the subjects. An improvement in walking ability was observed in patients suffering from peripheral arterial occlusive disease without previous unilateral lower limb amputation. Ischemic limb ulcers improved in 28/30 patients: a complete healing of the ischemic ulcers was obtained in 24 patients.

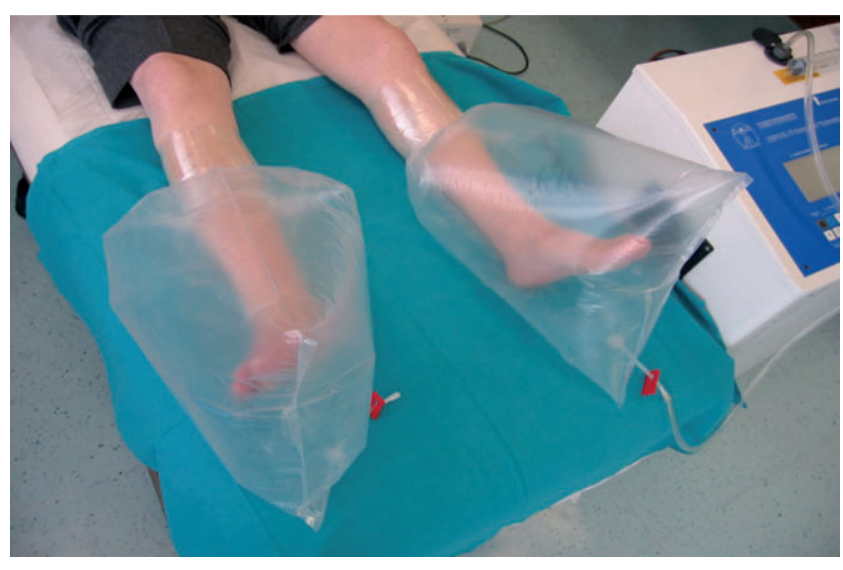

Figure 1. Ischemic leg exposure to ozone by bagging.

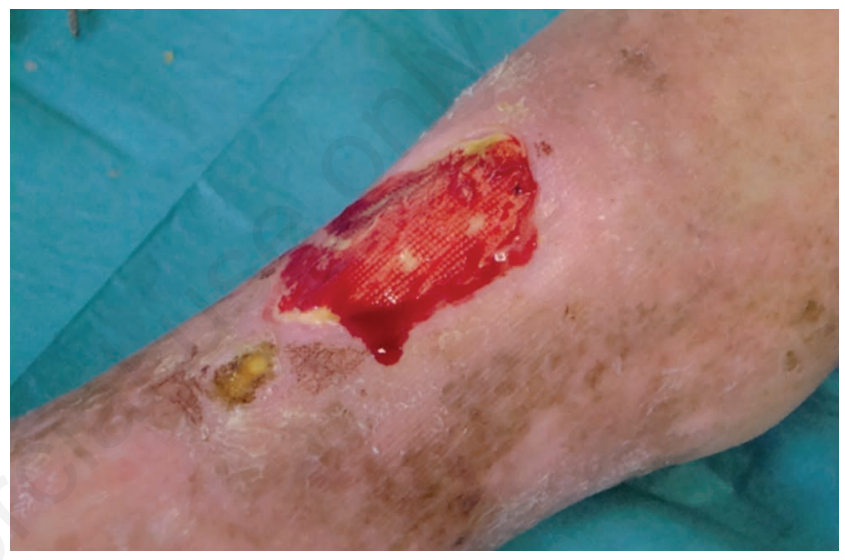

Figure 2. Very severe peripheral ulcerative lesions medicated with patient's own blood mixed with ozone (minor $\mathrm{O}_{3}$ autohemotherapy).

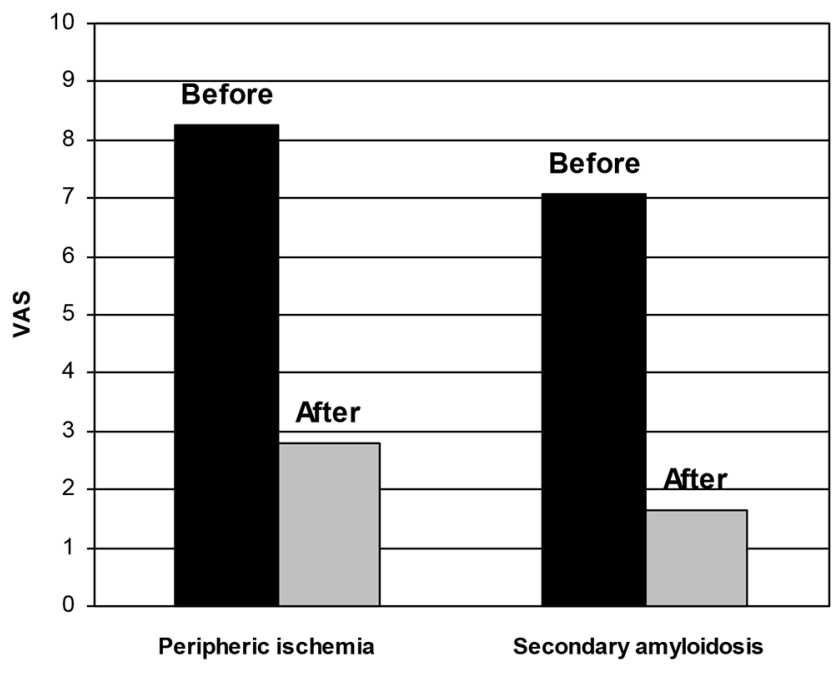

Figure 3. Visual analogic scale (VAS) before and after oxygenozone therapy. 


\section{Discussion and Conclusions}

In the past decade, a rapid growth in the number of older patients (over 65 years of age) requiring renal replacement therapy has been reported. Both age and chronic renal failure are risk factors for peripheral vascular disease and joint pain (due to arthritis and a2-microglobulin deposition respectively). Vascular nephropathy represents the major cause of end-stage renal disease, followed by diabetes. The death causes are mainly and the principal prognostic factors are the rate and the severity of cardiovascular comorbidities. Vascular disease and amyloidotic arthropathy represent two of the most critical aspects of dialysis in the elderly ${ }^{13,14}$ and contribute to strongly impair patient's quality of life by reducing walking and exercise ability.

Peripheral arterial occlusive disease accounts for significant morbidity and mortality among end-stage renal disease patients. Its prevalence appears to be much higher among uremic patients than in the general population and it is probably related to the effect of conventional, dialysis and uremia-associated risk factors.

Although peripheral arterial occlusive disease is a common disease in uremic patients, most of them are not screened for peripheral arterial disease; moreover prevention by smoking cessation, preventive foot care and exercise are not systematically applied to these subjects. ${ }^{8}$

Amputation is more common in hemodialysed patients than in the general population. Male sex, diabetes, previous diagnosis of peripheral vascular disease, mean systolic blood pressure and elevated serum phosphorous level are associated with the outcome of amputation; among patients without diabetes, a previous diagnosis of cardiac disease, a long history of dialysis therapy and previous hospitalization for limb ischemia are associated with an increased risk for future amputation. ${ }^{10}$ Thus the importance of preventing amputation in this population cannot be overemphasized and nephrologists should be aware of the remarkable value of detecting peripheral vascular disease by the screening of dialysed patients. The optimal management of ischemic ulceration and gangrene in end-stage renal disease patients is quite controversial. The management of peripheral arterial occlusive disease includes drug therapies, limb-sparing procedures such as percutaneous angioplasty and vascular reconstruction. Medications show little verified benefit. ${ }^{15,16}$ Diffuse distal lesions and vascular calcification in dialysed patients often impair the angioplasty success; moreover serious comorbid conditions make hemodialysed patients poor candidates for surgical procedures and contribute to increase mortality rate for those who undergo limb-sparing procedures. ${ }^{8}$ Hence non-invasive therapeutic procedures are desirable for this class of patients.

Dialysis-related amyloidosis secondary to a2-microglobulin deposits is a common complication of dialysis. It is preferentially located in the osteoarticular tissues, particularly in large bones close to joint spaces and synovial membranes although small deposits are also found in various organs, mainly the heart and gastrointestinal tract. Pathologic studies have demonstrated a high prevalence of articular a2-microglobulin early in the course of hemodialysis and peritoneal dialysis, preceding the clinical manifestations by several years and positively correlating to the time from the initiation of dialysis therapy. The key factor in the pathogenesis is the retention of a2-microglobulin associated with secondary modifications of the molecule such as limited proteolysis, conformational changes and the formation of advanced glycation end products. ${ }^{17}$ The biocompatible membranes for dialysis remove and adsorb a2-microglobulin more efficiently than the cellulosic membranes, but they are not enough to prevent the disease. Clinical manifestations are likely associated with the inflammation observed when the deposits involve capsules and sinovia with macrophage recruitment around the deposits. ${ }^{9}$ Patients develop carpal tunnel syndrome, remarkable pain in the shoulders, cervical and lumbar column. The management of secondary amyloidosis mainly consists in analgesia, leading to a high risk of gastro enteric bleeding.

Several papers have confirmed the beneficial effect of oxygenozone therapy in the treatment of vascular disorders, ${ }^{1-3}$ osteoarthritis, ${ }^{4,5}$ orthopedic pathology ${ }^{6}$ and various painful syndromes. ${ }^{4,5}$ Since peripheral arterial disease and secondary amyloidosis account for significant morbidity among end-stage renal disease patients, we performed a prospective study concerning the effect of ozone therapy on peripheral arterial disease and bone pain in hemodialysed patients.

Ozone therapy is commonly performed through minor $\mathrm{O}_{3}$ autohemotherapy and major $\mathrm{O}_{3}$ autohemotherapy; ${ }^{7}$ other medical uses include extracorporeal circulation, rectal insufflation and almosttotal body exposure. ${ }^{5}$ Topical applications include ozonated oil ${ }^{18}$ or water ${ }^{5}$ and intrarticular applications. ${ }^{6}$ Ozone is known to be a strong oxidant and when dissolved in the blood, it produces a number of reactive oxygen species (ROS) that are almost completely quenched by antioxidant systems present in the plasma and blood cells ${ }^{19}$ at a safe range of ozone concentration. Some ROS react with polyunsaturated fatty acids and generate hydrogen peroxide and lipid oxidation products; the latter is an important messenger responsible for transmitting the beneficial effects of ozone. Ozone effects include glycolysis activation and improvement of ATP levels, a slight induction in cytokins production and increased NO production; ${ }^{5}$ the increase in 2,3 -diphosphoglycerate levels (responsible for enhanced oxygen delivery to ischemic tissues) is still controversial. ${ }^{20}$ The beneficial effect of ozone therapy on peripheral ischemic disease may be due to a group of mechanisms that improve blood flow in hypoxic areas and decrease the symptoms of ischemia: decrease in blood viscosity and coagulation ${ }^{20,21}$ vasorelaxation ${ }^{22}$ and alterations in prostanoid production. ${ }^{23}$ Regarding osteoarticular pain, subcutaneous ozone infusion in specific muscle trigger points induces noxious inhibitory control and subsequent muscle relaxation, pain reduction and vasodilatation..$^{5}$ The therapeutic ozone dose ranges from 20 to $50 \mathrm{mcg} / \mathrm{mL}$ per gram of blood. The induction of oxidative stress represents a major problem concerning its application in medicine. Hemodialysed patients are known to be particularly exposed to the formation and deleterious effects of free radicals, due to

Table 1. Secondary amyloidosis and peripheral ischemia in hemodialysed patients: clinical results after ozone therapy.

\begin{tabular}{lcccc} 
& $\begin{array}{c}\text { Secondary } \\
\text { amyloidosis }\end{array}$ & $\begin{array}{c}\text { Peripheral ischemia } \\
\text { Ilb stage }\end{array}$ & $\begin{array}{c}\text { Peripheral ischemia } \\
\text { III stage }\end{array}$ & $\begin{array}{c}\text { Peripheral ischemia } \\
\text { IV stage }\end{array}$ \\
Optimal & 8 & 22 & 6 & 12 \\
Significant & 5 & 7 & 4 & 3 \\
\hline No change & 2 & 1 & 2 & 3 \\
Total & 15 & 30 & 12 & 18 \\
\hline
\end{tabular}


uremia and dialysis procedures. ${ }^{24}$ Oxidative stress is involved in several disease complications such as secondary amyloidosis and atherosclerosis. ${ }^{25,26}$ As a result an objection may be that ozone extends the oxidative stress even at low doses. The influence of ozone therapy on oxidative stress in hemodialysed patients has already been studied. Tylicki et al. demonstrated that ozonated autohemotherapy with ozone concentration of $50 \mathrm{mcg} / \mathrm{mL}$ per gram of blood, applied three times a week, induces no oxidative cell injury in the hemodialysed population: the antioxidant defence system neutralizes the oxidative properties of ozone at this concentration and protects against oxidative cell damage. ${ }^{2}$ Therefore ozone therapy is a safe method and may be a complementary clinical approach in hemodialysed patients when correct therapeutic doses are applied. In the present paper a subjective clinical improvement was reported by the majority of patients. A placebo effect, at least in part, may have played a role in limiting this measure based on subjective information; nevertheless clinical observations and the absence of changes in factors able to affect patients' outcome (hemodialysis adequacy, anemia) eliminate such a theory. Complete healing of the ischemic ulcers was obtained in a high percentage of patients. Individual decrease in pain perception was strengthened by analgesic dosage reduction or suspension. Bone pain perception and clinical signs of peripheral vascular disease reduction were observed. Subsequent achievement of normal sleep/wake cycle and better physical activity improved patients' quality of life. The concomitant reduction in analgesic use lowered the risk of gastro enteric bleeding, a frequent complication in dialysis patients. The result was obtained by a non-invasive therapeutic procedure; since serious comorbid conditions make hemodialysed patients poor candidates for surgical procedures, ozonotherapy may become a desirable therapeutic option for this class of patients. The present study, although uncontrolled, demonstrates the beneficial clinical effect of ozonotherapy in patients on undergoing hemodialysis. However given the small sample size and design limitations, further large-scale clinical randomized trials should be performed to give evidence to the clinical practice and to confirm the long-term beneficial effects of this therapy.

\section{References}

1. Valdes RA, Cepero MS, Moraleda GM, Pozo LJ. Ozone therapy in the advanced stages of arteriosclerosis obliterans. Angiologia 1993;45:146-8.

2. Tylicki L, Nieweglowski T, Biedunkiewicz B, et al. The influence of ozonated auto-hemotherapy on oxidative stress in hemodialysed patients with atherosclerotic ischemia of lower limbs. Int J Artif Organs 2003;26:297-303.

3. Bocci V, Zanardi I, Travagli V. Ozone: a new therapeutic agent in vascular diseases Am J Cardiovasc Drugs 2011;11: 73-82.

4. Bocci V. Oxygen-ozone therapy. A critical evaluation. London: Kluwer; 2002.

5. Di Paolo N, Bocci V. EBOO (Extracorporeal Blood Oxygenation and Ozonization). Cosenza, Italy: Bios; 2003.

6. Andreula CF, Simonetti L, De Santis F, et al. Minimally invasive oxygen-ozone therapy for lumbar herniation. Am J Neurorad 2003;24:784-7.
7. Wolff HH. Die behandlung perripherer durchblutungsstorungen mit ozon. Erfahr Hr 1974;23:181-4.

8. O'Hare A, Johansen K. Lower-extremity peripheral arterial disease among patients with end-stage renal disease. J Am Soc Nephrol 2001;12:2838-47.

9. Jadoul M, Garbar C, van Ypersele de Strihou C. Pathological aspects of $\beta 2$-microglobulin amyloidosis. Semin Dial 2001; $14: 86-9$.

10. O'Hare AM, Bacchetti P, Segal M, et al. Factors associated with future amputation among patients undergoing hemodialysis: results from the Dialysis Morbidity and Mortality Study Waves 3 and 4. Am J Kidney Dis 2003;41:162-70.

11. Daugirdas JT. Second generation logarithmic estimates of single-pool variable volume Kt/V: an analysis of error. J Am Soc Nephrol 1993;4:1205-13.

12. Italian Society for Oxygen-Ozone Therapy. SIOOT Protocols. Gorle (BG), Italy: SIOOT; REV.01.14.192 2003. Available from: www.ossigenoozono.it

13. Società Italiana di Nefrologia. Italian Registry of Dialysis and Transplantation. Report 2012. Rome, Italy: SIN; 2012. Available from: www.sin-italy.org/sin-ridt/

14. Alloatti S, Manes M, Gaiter AM, et al. Dialysis in the elderly. G Ital Nefrol 2002;19:622-9.

15. Creutzig A, Creutzig H, Alexander K. Effects of intra-arterial prostaglandin E1 in patients with peripheral arterial occlusive disease. Eur J Clin Invest 1986;16:480-5.

16. Money S, Herd J, Isaacsohn J, et al. Effect of cilostazol on walking distances in patients with intermittent claudication caused by peripheral vascular disease. J Vasc Surg 1998;27:267-74.

17. Floege J, Ketteler M. Beta 2-microglobulin-derived amyloidosis: an update. Kidney Int Suppl 2001;78:S164-71.

18. Menendez S, Falcon L, Simon DR, Landa N. Efficacy of ozonized sunflower oil in the treatment of tinea pedis. Mycoses 2002;45:329-32.

19. Bocci V. Biological and clinical effects of ozone. Has ozone therapy a future in medicine? Br J Biomed Sci 1999;56:270-9.

20. Giunta R, Coppola A, Luongo C, et al. Ozonized autohemotransfusion improves hemorheological parameters and oxygen delivery to tissues in patients with peripheral occlusive arterial disease. Ann Hematol 2001;80:745-8.

21. Sroczynski J, Antoszewski Z, Krupa G, et al. Some hemostatic parameters after treatment with ozone in a group of patients with obliterative atherosclerosis of the lower extremities and in a group of patients with diabetes. Pol Tyg Lek 1991;46:694-6.

22. Dutka M, Adamczak M, Kopieczna, et al. Vasorelaxant activity of ozone in vitro studies. Adv Clin Exp Med 1998;7:391-8.

23. Bodzenta E, Bujak E. Level of certain prostanoids in serum of patients treated with ozone injected intra-arterially. Pneumonol Allergol Pol 1994;62:S77-82.

24. Morena M, Cristol JP, Canaud B. Why hemodialysis patients are in a prooxidant state? What could be done to correct the pro/antioxidant imbalance. Blood Purif 2000;18:191-9.

25. Iuliano L. The oxidant stress hypothesis of atherogenesis. Lipids 2001;36:S41-4.

26. Miyata T, Notoya K, Yoshida K, et al. Advanced glycation end products enhance osteoclast-induced bone resorption in cultured mouse unfractioned bone cells and in rats implanted subcutaneously with devitalized bone particles. J Am Soc Nephrol 1997;8:260-70. 